\title{
Detection of Leucocytes in Microscopic Images with Swarm Intelligence Algorithm
}

\author{
${ }^{1}$ M. Venkata Dasu, ${ }^{2}$ Dr.P.Subbaiah \\ ${ }^{1}$ Research Scholar, ${ }^{2}$ Professor \\ ${ }^{* 1}$ Department of ECE, Rayalaseema University, Kurnool, Andhra Pradesh., India \\ ${ }^{2}$ Department of ECE, Nalla Narasimha Reddy Engineering College, Ghatkesar, Hyderabad, Telangana., India \\ Email: ${ }^{1}$ dassmarri@gmail.com, ${ }^{2}$ subbaiah.nani45@gmail.com
}

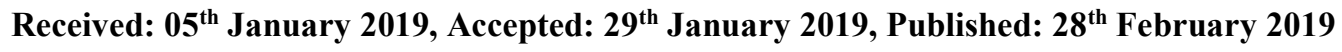

\begin{abstract}
Leukemia is a blood a cancer. It is also Known as Hematologic cancer. Leukemia is due to abnormal growth of blood cells by the bone marrow in human body. It normally affects the white blood cells (Leucocytes). This disease can be recognized and diagnosed by analyzing microscopic images. Generally blood cells are captured by microscopic images. Early detection of cancer increases the survival rate of the patient. To detect leukemia cells in blood smear hematologists finds difficulty and it is inaccurate and time consuming. So it requires fast and cost effective system to identify the disease. Image processing techniques are most useful for the analysis of microscopic images. These methods do not require special equipment for lab testing .Image processing techniques are fast and cheap. In this paper Image processing methods and optimization algorithms are used for the detection of leucocytes. ACO (Ant colony optimization) and PSO (Particle swarm optimization algorithm) are existing algorithms and a Cuckoo search Optimization algorithm is proposed method (CSO). By observing Statistical parameters the proposed method outperforms the existing methods. These results are obtained by simulating MATLAB software with I5 processor and 8GB RAM.
\end{abstract}

\section{Keywords}

Enhancement; Median Filter; Segmentation; Leukemia: Optimization

\section{Introduction}

As of late, Digital image processing is connected to numerous applications which incorporate in remote sensing, biomedical and so on. Specifically, imaging applications are rising as another open door for modernization at the meeting point among computer science and medicine. Image processing is valuable to remove significant data from medicinal images and enhances the diagnosis of Leukemia in patients [1]. Malignant cells can be detected by human look and it takes lot of time and inaccurate also .Image processing plays important step in providing the correct form of diagnosis.

Leukemia is the most dangerous disease, it can progress rapidly and if the patient not treated in time it may leads to fatal death within few months. Leukemia is mostly effects in children and adults. Generally majority malignancy cells start in body parts yet leukemia is the sort of disease which starts and develops in blood cells (1). Blood is essential substance without which metabolic elements of body is seriously influences. Bone marrow produces Blood cells. Blood cell comprises of three distinct kinds of cells such as WBC, RBC and Platelets. Erythrocytes (RBCs) are accountable for taking oxygen and CO2 away from tissues. There are three kinds of WBCs they are called as lymphocytes, monocytes, eosinophils, basophils and neutrophils [2-3]. Typically, leucocytes (WBCs) combat with the foreign bodies and keeps from contamination. Platelets help the coagulating of blood and controls hemorrhage (2)

In Human system cell grows and multiply into new cells. In cancer disease, the old cells are not perished and remains in the blood. So the newly generated cells can't have sufficient space to live and finally the functioning of blood distracts and generation of blood cells is irregular and uncontrolled [4].

In this work a novel method (Cuckoo Search algorithm) is proposed for the detection of cancerous (Malignant) cells accurately in microscopic medical images. Also in this work the obtained values from the proposed method are compared with the existing methods (ACO \& PSO).

\section{Literature Review}

In 2017 DessaiVihangiHiren et.al [5] used fuzzy system for accurately separates the overlapped leukocytes (white blood cells) from blood samples. In Pre-Processing they have used morphological hole filling and contrast enhancement is used. In this work the author obtained segmentation accuracy of $94.07 \%$.

In 2017 Afaftareef et.al [6] proposed watershed technique for segmentation of leukocytes (white blood cell). In pre-processing morphological operations are used for image enhancement.

In 2016 Jullendgetc et.al [7] used double thresholding and binary large objecy (BLOB) method for classification of blood cells. In this work they obtained sensitivity $84.43 \%$ and Specificity $85.5 \%$. 
In 2015 Shubhangi Hhobragads et.al [8] used a Otsu thresholding technique for segmentation and feature extraction is implemented on LABVIEW. In pre-processing histogram equalization is used for enhancement. In this work the main statistical parameters are considered for detection of Leukemia are mean and standard deviation.

In 2015 S.S.Savkare et.al [9] used K-means clustering technique for segmentation of leukocytes in microscopic images and laplacian filter is adopted for image enhancement in pre-processing step. In this work they have obtained segmentation accuracy of $95.5 \%$.

In 2014 Nanly.N.salemet.al [10] authors converted microscopic RGB image in to L*a*b color space and segmentation is carried out by K-means clustering algorithm.

In above all the proposed methods authors have got less segmentation accuracy because the extraction of overlapping blood cells is difficult. In this work Optimization algorithms are proposed for accurate segmentation of leucocytes in blood samples and scale invariant feature transform is used for feature extraction.

\section{Methodology}

The proposed model consists of image processing and optimization techniques as shown in fig.1.These techniques are used to detect cancerous white blood cells in microscopic images. Optimization means maximizing or minimizing objective function by choosing inputs systematically from the available set.

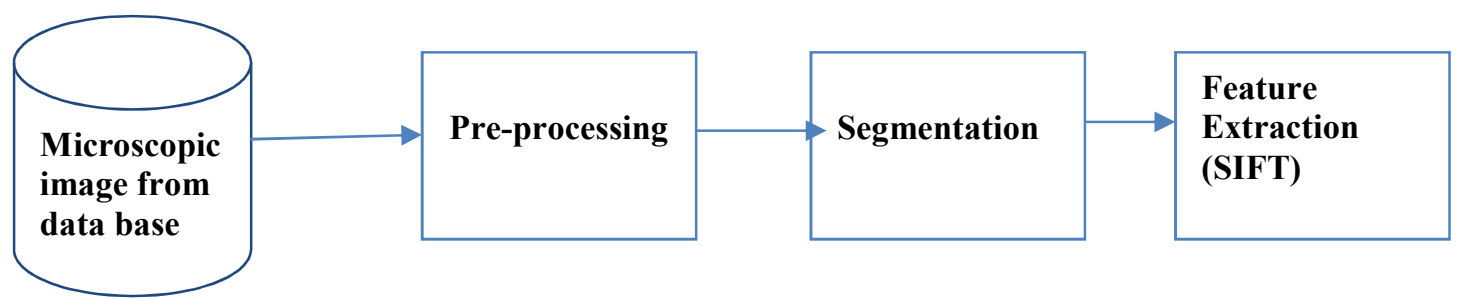

Fig. 1: Proposed Block Diagram.

The proposed model contains the following steps.

Algorithm Used for Detection for Cancer Cell:

Step 1: Acquiring of Input microscopic image.

Step 2: RGB image to gray scale conversion

Step 3: Removal of noise using median filter

Step 4: Contrast enhancement can be achieved through CLAHE method

Step 5: Apply Optimization algorithms for segmentation of cancer cells.

Steps6: Features of segmented cell can be obtained using SIFT (Scale invariant feature transform).

\section{A. ACQUISITION OF INPUT IMAGE}

It is the initial stage in the many vision system. Microscopic images are generally acquired from pubic data base or from private data base. In this work images are obtained from public data base such as Microscopyu.

\section{B. PREPROCESSING}

In this work Preprocessing contains color conversion and noise removal and Image Enhancement.

- RGB to Gray Scale Conversion: Digital microscopes generate the RGB color space images. Direct processing RGB image is difficult because image back ground and blood cells varies with color and intensity. It may be due to several reasons like varying illumination, camera settings etc. So to overwhelmed this issue primarily the color image (RGB) image is converted in to gray scale.

- Noise Removal: In Digital image processing system removal of noise is the most challenging problem. In this work an efficient method (Median filter) is used for noise reduction. This filter also eliminates the degradation function and corrupted noise components [11]. This filter is a nonlinear and designed for a desired frequency response. It reduces the MSE (mean square error).

- Image Enhancement: Image Enhancement can be widely used for many image processing applications where the subjective quality of an image can be improved for human visual perception. Contrast is an important parameter and makes the particular object recognizable from the image which contains more objects and background. It is determined based on the difference of brightness and of the color two objects. Various procedures such as HE and AHE were established and applied to the several issues related to image processing applications. Each procedure has their own limitations. AHE (Adaptive Histogram Equalization) over amplifies the noise. So in this work CLAHE (Contrast Limited Adaptive histogram) is utilized which lessens the over enhancement of noise by section the histogram at a predefined esteem (called clip limit) before calculating the CDF. Also the edges of white blood cells become keener by using CLAHE [12]. 


\section{SEGMENTATION}

The fundamental point of the image segmentation is to segment an image into numerous parts. It is a very important area of research in digital image processing. There is a lot of empirical research carried out by many researchers on different Digital image processing applications and approaches. Several algorithms are accessible for segmentation. However one single algorithm is not suitable for various images. Image processing application requires a segmentation algorithm which gives accurate segmentation result and less processing time. In this paper optimization algorithms are used for segmentation of cancerous WBCs (white blood cells) from microscopic images [13-15].Existing algorithms are ACO, PSO and proposed algorithms is CSO.

\section{Segmentation by Optimization Algorithms}

D. ANT COLONY OPTIMIZATION (ACO):

ACO is a heuristic optimization method utilized to solve the problems of discrete optimization. It is depends on the natural behavior of the ants they finds optimum paths between their nest and food[16].Ants will search for food by laying down pheromone trails and shortest path will discovered by the other ants via pheromone trails.

\section{ACO algorithm}

- Generate 'n' solutions randomly

- For individual ant A,

Choose each variable for a selected value $A=1,2, \ldots \mathrm{m}$

- Evaluate objective function

- Obtain good solution by changing the concentration of pheromone

$\rho_{x y}(i)=C \rho_{x y}(i-1)+\Delta \tau_{x y} \quad$ where $i=1,2 \ldots \ldots . M$

$\mathrm{M}$ is no. of iterations

$\rho_{x y}(i)$ concentration of pheromone at $i^{\text {th }}$ iteration

$\rho_{x y}(i-1)$ concentration of pheromone at (i-1) iteration

$\mathrm{C}$ is pheromone evaporation rate it's value ranges between 0 to 1

The drawback of ACO is if the search space is larger it takes longer time to converge the solution.

\section{E. PARTICLE SWARM OPTIMIZATION (PSO):}

It is a stochastic optimization technique based on population. The PSO was founded in 1995 by Dr. Eberhart and Dr. Kennedy evolved, inspired by the social behavior of the flock of birds or fish schooling. PSO finds approximate solutions for the extremely difficult numerical maximization and minimization problems. It is a simple and can be easily implemented for several optimization problems. In this algorithm when particle is moving from one place to another place it compares the previous place is nearer to the food or current place is nearer to the food and it also takes the knowledge of neighboring particle also [17].

Algorithm

- Initialize the particle with random motion \& velocity vector

- Evaluate fitness (P)

- If $\mathrm{P}$ better than the $\mathrm{P}_{\text {best }}$

- Set finest value of $P_{\text {best }}$ as $\mathrm{G}_{\text {best }}$

- Update velocity and position of particle

- Stop: giving $\mathrm{G}_{\text {best }}$ (optimal solution)

The velocity update equation $v_{\mathrm{i}}(\mathrm{i}+1)=\mathrm{w} \cdot \mathrm{v}_{\mathrm{i}}(\mathrm{i})+\mathrm{r}_{1}\left(\rho_{\mathrm{i}}(\mathrm{t})-\mathrm{y}_{\mathrm{i}}(\mathrm{t})\right)+\operatorname{ar}_{2}\left(\mathrm{~s}(\mathrm{t})-\mathrm{y}_{\mathrm{i}}(\mathrm{t})\right.$

Where $\mathrm{w}$ is called inertial weight and ranges between 0 to 1 .

PSO has drawback that it requires longer computational time than mathematical approaches

\section{F. CUCKOO SEARCH OPTIMIZATION (CSO):}

It is an exhaustive optimization algorithm depends on the behavior of the cuckoo bird. It was developed by Yang and Deb in 2009.CSO is established on the main idea of laying the eggs in host nests. If the eggs are not detected or destroyed the eggs are hatched to chicks by the hosts. Moreover, compared to host eggs the cuckoo eggs are hatched earlier [18-19].

Algorithm

1. Generate $\mathrm{n}$ hosts nests

2. Lay the egg $\left(a_{k}^{\prime}, b_{k}{ }^{\prime}\right)$ in the k nest, Where $a_{k}=a_{k}+$ randomwalk (levy flight) $a_{k}$ $b_{k}{ }^{\prime}=b_{k}+$ randomwalk (levy flight) $b_{k}$

3. Compare the fitness of Cuckoo's egg with fitness of host egg

4. If fitness of Cuckoo's eggs better than host eggs replace the in nest $\mathrm{k}$ by Cuckoo's eggs

5. If host bird notice it the nest is abandoned and new one will built

6. Till the termination condition is satisfied repeat steps 2 to 5

\section{G. SIFT (SCALE INVARIANT FEATURE TRANSFORM):}

In this work features of segmented white blood cell can be extracted by Scale Invariant Feature Transform.

Algorithm

1. Finds the characteristic scale for feature (detection of scale-space extrema) 
2. Select the key point based on a measurable stability(localization of key point)

3. Computes finest orientation for every key point region(Assignment of orientation)

4. Select image gradient and rotation at chosen scale to define every region of key point (Description of key point)

\section{Results \& Discussions}

In this work microscopic images are considered from public database Micoscopyu. In biomedical images processing starts with preprocessing.

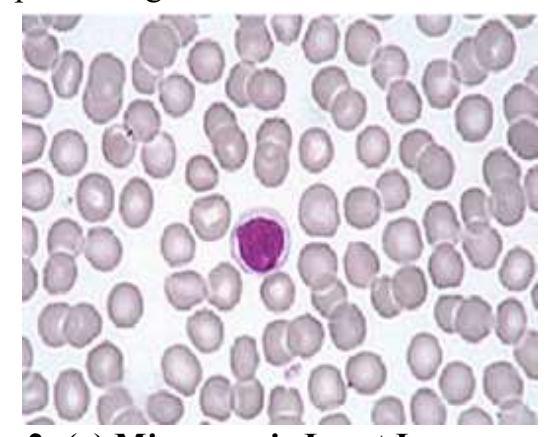

Fig.2: (a) Microscopic Input Image

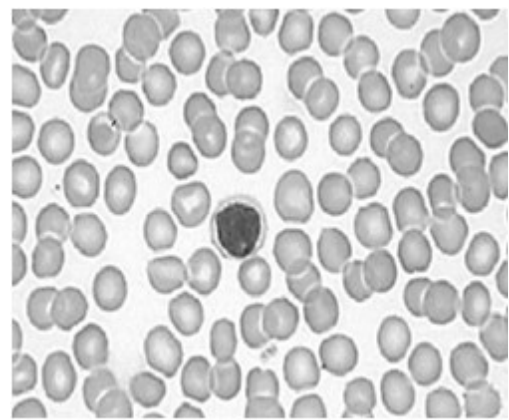

(b) Median Filtered Image

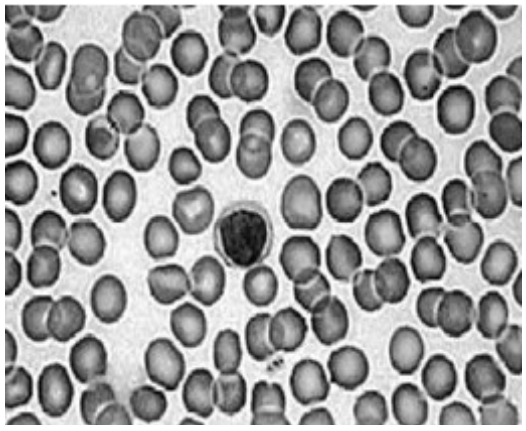

(c) CLAHE Image

Acquired microscopic images contain undesired and transient pixels along with noise. These can be removed by median filter. The input image is shown in fig. 2(a) figure (b) shows the median filtered image and (c) shows the contrast enhancement image using CLAHE method. It makes the boundaries of leucocytes (white blood cells) sharper.

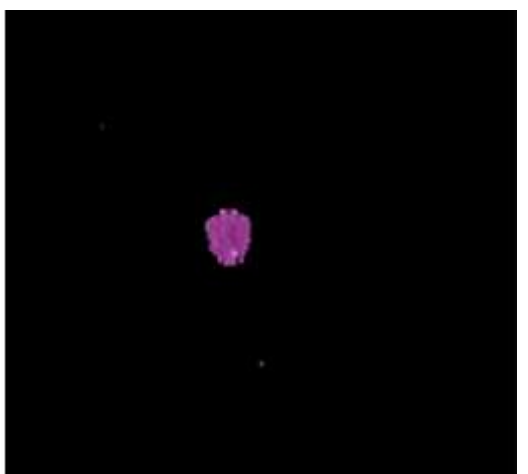

Fig.3: (a) Segmented Image using ACO

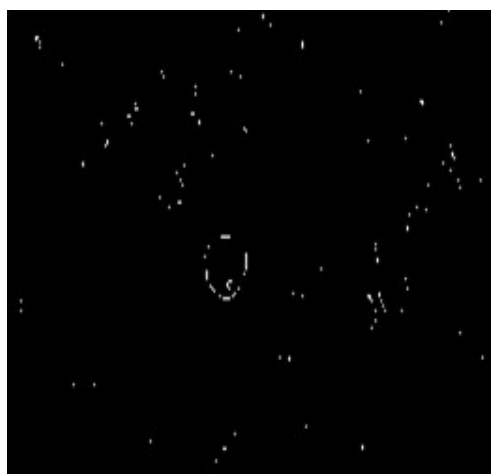

(b) Segmented Image using PSO

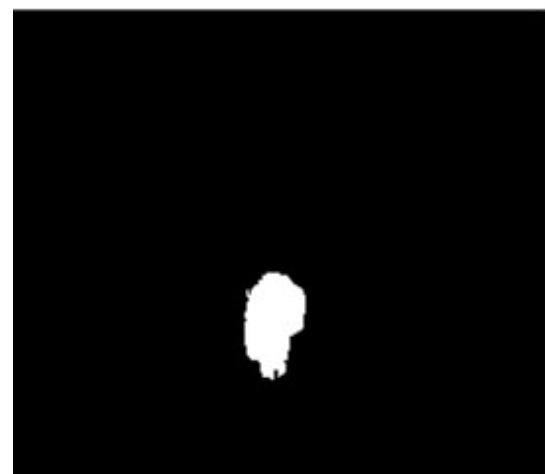

(c) Segmented Image using CSO

In blood samples cells gets deformed to arbitrary shapes due to environmental pressures. The cells may overlap with the neighboring cells. Segmentation overcomes this problem of overlapping. Fig.3. (a), (b) and (c) shows the segmented images using existing methods and proposed method. The segmented cell with CSO has sharper edges and is having better visual interpretation. Segmented cell features can be extracted by SIFT (Scale Invariant Feature Transform) technique. Table 1 shows the performance parameters like PSNR, Accuracy, Sensitivity, Specificity, FPR and CPU time. The proposed method has obtained better values than existing methods. Figure 4 shows the graphical representation of obtained values.

\begin{tabular}{|c|c|c|c|c|}
\hline S. No & Parameters & ACO & PSO & CSO \\
\hline 1 & PSNR & 22.90 & 23.34 & 24.16 \\
\hline 2 & ACCURACY & 20.58 & 56.18 & 97.58 \\
\hline 3 & SENSITIVITY & 28.03 & 48.34 & 89.32 \\
\hline 4 & SPECIFICITY & 29.04 & 46.15 & 90.72 \\
\hline 5 & FPR & 47.79 & 44.5 & 42.79 \\
\hline 6 & CPU Time (sec) & 15 & 10 & 8 \\
\hline
\end{tabular}

TABLE I: Obtained Parameters 
Helix Vol. 9 (1): 4838- 4842

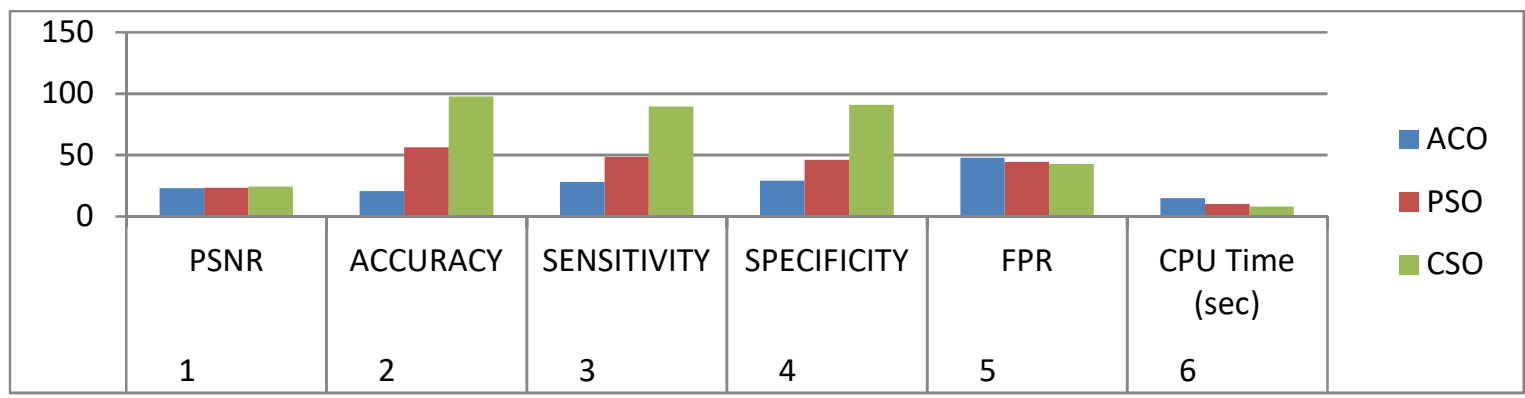

Fig.4: Graphical Representation of Obtained Parameters.

\section{Conclusion}

In this work, a heuristic optimization algorithm is adopted to identify the cancerous cells in microscopic blood smear. The preprocessing techniques in proposed model are median filter and CLAHE (Contrast Limited Adaptive Histogram Equalization) method. The segmentation accuracy of the proposed method Cuckoo search algorithm is $97.58 \%$ is higher value than proposed methods. The advantage of proposed method is very accurate and less time consuming.

\section{Acknowledgment}

The First author express sincere thanks to Rayalaseema University, Kurnool, Andhra Pradesh, where he is a research scholar and AITS, Rajampet, A.P. for providing good research facilities.

\section{References}

[1] RakibulAhasan,"white blood cells nucleus segmentation from microscopic images of strained peripheral blood film during Luekemia and normal conditions" in International conference on informatics,2016,Pg.No:361-366

[2] M. Veluchamy, K. Perumal, and T. Ponuchamy, "Feature extraction and classification of blood cells using artificial neural network," American Journal of Applied Science, vol. 9, no. 5, 2012, Pg.No. 615-619

[3] F. Kasimn, A. S. Prabuwono, and A. Abdullah, "Detection of leukemia in human blood sample based on microscopic images: a study," Journal of Theoretical and Applied Information Technology, vol. 46, no. 2,2012

[4] F. Jabar, W. Ismail, R.A. Salam, R. Hassan, "Image segmentation using a hybrid clustering technique and mean shift for automated detection acute leukemia blood cells images", Journal of Theoretical and Applied Information Technology, vol. 76, 2015, pp 88-96.

[5] Desai Vihangi Hiren,NishaVilani” An automated blood cell segmentation using Fuzzy Based system,IEEE,2017.

[6] AfafTareef,Yang song," Automated multistage segmentation of white blood cells via optimizing color processing",IEEE,2017,P.No:565-568.

[7] Jullendgatc, Febrimaspriyanti," Red blood cell and white blood cell classification using Double thresholding and BLBO analysis" in international conference on information and communication technologies,2016.

[8] Subhangi Khobragade, Dheeraj,"Detection of Leukemia in microscopic white blood cell images", in International conference on information processing,2015,P.no :435-440.

[9] S.S.Savkare,S.P.Narote,'Blood cell segmentation from microscopic blood images", in international conference on information processing,2015,P.No:502-505.

[10] Nancy.N.salem,"Segmentation of white blood cells from microscopic images using k-means clustering" in National radio conference, 2014,P.No:371-376.

[11] Sivappriya T ,Muthukumaran K, “Cancer Cell Detection Using Mathematical Morphology”, International Journal of Innovative Research in Computer and Communication Engineering, Vol.2, Special Issue 1, March 2014.

[12]I., Cseke, “A Fast Segmentation Scheme for white Blood Cell Images”, IEEE, 1992, Pg. No: 530-533

[13] M.Saritha,"Detection of blood cancer in microscopic images of human blood samples", International conference on electrical, electronics and optimization techniques,2016,Pg.No:596-600

[14]N., Ritter, J., Cooper, "Segmentation and Border Identification of Cells in Images of Peripheral Blood Smear Slides", 30thAustralasian Computer

Science Conference, Conference in Research and Practice in Information Technology, Vol. 62, 2007, Pg.No.161-169.

[15] Bhagyashri G Patil, Prof. SanjeevN.Jain, "Cancer Cells Detection Using Digital Image Processing Methods" in International Journal of Latest Trends in Engineering and Technology.https:/www.ics.uci.edu/ welling/teaching/271fall09/ant colonyopt.pdf

[16] Kennedy, J., R. C. Eberhart, and S. Yuhui. Swarm Intelligence. Morgan Kaufmann, San Francisco, 2001.

[17] Xin-She Yang, Suash Deb, "Cuckoo Search via L'evy Flights", IEEE, 2009

[18] Karaboga D., Ozturk C., 2011, "A novel clustering approach: Artificial Bee Colony (ABC) algorithm", Elsevier B.V. journal for Applied Soft Computing (652-657). 